\title{
Tendencia nacional de la mortalidad por hepatocarcinoma registrada en el Ministerio de Salud del Perú del 2005 al 2016
}

\author{
Alejandro Piscoya, ${ }^{1}$ Noé Atamari-Anahui, ${ }^{2}$ Maycol Suker Ccorahua-Rios, 3 Angela Parra \\ del Riego ${ }^{4}$ \\ ${ }^{1}$ Universidad San Ignacio de Loyola, Unidad de Revisiones Sistemáticas y Metaanálisis, Guias de Práctica Clínica y Evaluaciones de Tecnologías \\ Sanitarias. Lima, Perú. \\ ${ }^{2}$ Universidad San Ignacio de Loyola, Unidad de Investigación para la Generación y Sintesis de Evidencias en Salud. Lima, Perú. \\ ${ }^{3}$ Universidad Nacional de San Antonio Abad del Cusco, Facultad de Ciencias de la Salud, Escuela Profesional de Medicina Humana, Asociación \\ Cientifica de Estudiantes de Medicina Humana (ASOCIEMH CUSCO). Cusco, Perú. \\ ${ }^{4}$ Universidad Peruana de Ciencias Aplicadas, Facultad de Ciencias de la Salud, Escuela de Medicina, Lima, Perú.
}

Acta Gastroenterol Latinoam 2020;50(3):292-299

Recibido: 14/09/2019 / Aceptado: 12/08/2020 / Publicado online: 28/09/2020

\section{Resumen}

Antecedentes. El hepatocarcinoma resulta en la mayoría de los casos de una enfermedad hepática crónica subyacente. Las causas más comunes son la infección por el VHB y VHC, el alcoholismo y la aflatoxina. La estadistica sobre la mortalidad del hepatocarcinoma en Perú es limitada. Objetivos. Actualizar la estadistica sobre la mortalidad de hepatocarcinoma en el Perú entre los años 2005 y 2016. Métodos. Estudio observacional, descriptivo y análisis secundario de la base de datos del MINSA. Se revisaron los registros con la causa básica de muerte CIE 10: C22, el carcinoma de células hepáticas. Se calculó la mortalidad según la edad, el sexo y el departamento en el que se registró la defunción; además, se computó la mortalidad estandarizada por edad.

Correspondencia: Alejandro Piscoya

Maestro Barbieri 182, San Borja (C.P.: 15037). Lima, Perú Tel.: 0051999703885

Correo electrónico: alepiscoya@gmail.com
Resultados. 2170 personas fallecieron por un hepatocarcinoma. El 50,1\% era varón y el 67,5\% mayor de 60 años. La tasa de mortalidad estandarizada en Perú disminuyó del 1,1 al 0,7 por cada 100.000 habitantes del 2005 al 2016. La tasa cruda de mortalidad por cada 100.000 habitantes muestra que comparando el primer periodo (20052010) con el segundo (2011-2016), la tendencia en Perú es a disminuir. La única región que presentó disminución de la mortalidad fue la de la Sierra (\% de cambio = -40,1). Conclusiones. La mortalidad estandarizada por edad tuvo una ligera disminución desde el 2005 al 2016; no obstante, esta diferencia no muestra variaciones considerables. La mortalidad por esta neoplasia parece mantenerse elevada y estable desde el periodo de 1995 al 2000.

Palabras claves. Hepatocarcinoma, mortalidad, Perú.

\section{National Trends of hepatocellular carcinoma mortality registered by the Ministry of Health in Peru, from 2005 to 2016}

\section{Summary}

Background. Hepatocellular carcinoma results in most cases from underlying chronic liver disease. The most common 
causes are the Hepatitis B virus and the Hepatitis C virus infections, the alcoholism and the aflatoxin. Mortality statistics of liver cell carcinoma in Peru is limited. Objectives. Update statistics on hepatocellular carcinoma mortality in Peru between the years 2005 and 2016. Methods. Observational, descriptive studyand secondary analysis of the Ministry of Health database. Records with the basic cause of death ICD 10: C22, the liver cell carcinoma were reviewed. Mortality was calculated according to the age, the sex and the department in which death was recorded; Also, standardized mortality by age was calculated. Results. 2,170 people were registered as deceased due to hepatocellular carcinoma. The $50.1 \%$ were male and the $67.5 \%$ older than 60 years. The standardized mortality rate in Peru decreased from 1.1 to 0.7 per 100,000 population from 2005 to 2016. The raw cup of mortality per 100,000 population shows that when comparing the first period (2005-2010) with the second (2011-2016), the tendency in Peru has decreased. The only region that presented a decrease in mortality was the Mountains (\% change = -40.1). Conclusions. Standardized mortality by age had a slight decrease from 2005 to 2016; however, this difference does not show considerable variations. Mortality from this neoplasm seems to remain high and stable since the period from 1995 to 2000.

Key words. Hepatocellular, carcinoma, cause of death, Peru.

\section{Abreviaturas}

VHB: Virus de la hepatitis $B$.

VHC: Virus de la hepatitis $C$.

MINSA: Ministerio de Salud del Perú.

CIE 10: Clasificación Internacional de Enfermedades.

BCLC: Barcelona Clinic Liver Cancer.

EHGNA: Enfermedad por hígado graso no alcohólico.

ASMR: Age-standarized mortality rate.

TME: Tasa de mortalidad estandarizada.

\section{Introducción}

El hepatocarcinoma es el tumor primario del hígado más común y se origina en el 70 al $90 \%$ de los pacientes con una enfermedad hepática crónica. ${ }^{1}$ Hoy en día, es la sexta neoplasia más frecuente en el mundo (con 841080 nuevos casos diagnosticados en el 2018) y es la segunda causa de muerte por cáncer. ${ }^{2}$ Existen diferencias según el género, por lo que esta neoplasia escala al quinto lugar en los hombres y desciende al noveno lugar en las mujeres. Los países con mayor cantidad de casos se encuentran en Asia y África. Solo entre los años 2012 al 2018 ha habido un aumento de 59080 nuevos casos en el mundo. ${ }^{2}$

La mortalidad por esta neoplasia ha aumentado más de 2 veces en Estados Unidos (EE. UU.) desde 1980 donde el sexo masculino, la edad superior a 65 años y la raza (asiática, africanos americanos e hispanos) componen las principales categorías de mayor mortalidad. ${ }^{3}$

En Europa, el hepatocarcinoma es responsable de 47000 muertes anuales. ${ }^{4}$ Esto genera un importante problema de salud pública, no sólo por los grandes costos en salud, sino por los grandes años de vida perdidos y la carga por la enfermedad. ${ }^{3}$

La clasificación de la Barcelona Clinic Liver Cancer (BCLC) es uno de los sistemas de estadiaje del hepatocarcinoma para el tratamiento y el pronóstico sugeridos por los organismos internacionales como la Asociación Estadounidense para el Estudio de las Enfermedades del Hígado y la Asociación Europea para el Estudio del Hígado. En esta clasificación se especifica como la supervivencia en estadios muy tempranos y tempranos puede ser de más de 5 años mientras que, en los casos de cáncer intermedio, avanzado y terminal, la supervivencia suele ser de 2 a 5 años, 1 año y 3 meses respectivamente. ${ }^{5}$

En el Perú, el hepatocarcinoma ocupa el décimo tercer lugar de las neoplasias más frecuentes según las últimas estadísticas reportadas hasta el 2012. ${ }^{6}$ Además, el Instituto Nacional de Enfermedades Neoplásicas (INEN) informó, del 2012 al 2017, más de 1000 nuevos casos de hepatocarcinoma, lo cual representa un incremento de más del $60 \%$ con respecto a cifras del año 2000 (INEN, 2017)..$^{7}$ Al hablar de la mortalidad, el cáncer del hígado y de las vías biliares ocupa el segundo lugar con el 11\% (3362 casos) sólo por detrás del cáncer de estómago. ${ }^{8}$

Asimismo, durante los últimos años (2000-2011), la tasa ajustada de mortalidad por este cáncer no ha mostrado una variación significativa. Los departamentos de la Selva como los de Amazonas, Madre de Dios y Ucayali ocupan los primeros lugares de mortalidad en el 2011, seguidos de los departamentos de la Sierra como Apurímac, Ayacucho, y Huánuco. ${ }^{8}$

El hepatocarcinoma se origina en un $80 \%$ de los casos en una cirrosis hepática preexistente. ${ }^{1}$

Los factores predisponentes más importantes para desarrollar la cirrosis hepática varían de acuerdo con 
el continente, ya que, en Asia y África, predomina la infección por el VHB y la aflatoxina B1, mientras que, en Europa y Norteamérica, predomina la infección por el VHC y el alcohol. ${ }^{5} 9$ Otro factor importante, cada vez más común, es la la enfermedad de hígado graso no alcohólico. ${ }^{1}$ Además, existen cofactores como la diabetes mellitus, el síndrome metabólico y el tabaquismo que contribuyen al desarrollo del hepatocarcinoma en los pacientes con la EHGNA. ${ }^{5}$ En el Perú, la información es limitada. Sin embargo, el VHB fue el factor de riesgo más asociado al hepatocarcinoma en los dos estudios previos en los años 2007 y 2009. ${ }^{10,11}$

En nuestro país, la mortalidad por esta neoplasia ha ocupado puestos altos por más de veinte años, así como también se ha demostrado que la tendencia se mantiene sin ninguna variación considerable desde el año $2000 .{ }^{8} \mathrm{La}$ infección por el VHB sigue encabezando las principales causas de esta neoplasia a pesar de que la vacunación en los niños menores de un año comenzó en 1996 en las zonas hiperendémicas y en el 2003 de forma universal. ${ }^{12}$ Los estudios en los EE. UU. muestran una disminución de la infección por el VHB en un 68\% luego de 10 años del inicio de la vacunación universal. ${ }^{13}$

Perú aún presenta cifras que indican que no hay un impacto favorable sobre la cirrosis y el hepatocarcinoma asociados a la infección por el VHB, mostrando incluso que desde el 2002 hay una tendencia que está aumentando. ${ }^{14}$

Es por este motivo que hemos realizado un análisis de la mortalidad por el hepatocarcinoma entre los años 2005 al 2016 en nuestro país: para actualizar las cifras nacionales e indagar sobre el problema actual. De esta manera, buscamos poder impulsar nuevos estudios e intervenciones que logren mejorar la situación actual de esta patología.

\section{Métodos}

\section{Diseño de estudio y unidad de análisis}

Realizamos un estudio descriptivo y un análisis de datos secundarios. Las unidades de análisis fueron las 25 regiones políticas del Perú (24 departamentos y una provincia constitucional) y sus tres regiones geográficas (Costa, Sierra y Selva).

\section{Fuente de datos y procedimientos}

La información se obtuvo de los registros anuales de las defunciones como causa básica del Ministerio de Salud del Perú (MINSA).
Dicha información proviene de los certificados de defunción del periodo 2005-2016, para lo cual los solicitamos a través de la Plataforma de Acceso a la Información Pública (http://www.minsa.gob.pe/portada/transparencia/solicitud/frmFormulario.asp) mediante la codificación CIE 10: C22, carcinoma de células hepáticas.

\section{Variables}

La variable de interés fue la mortalidad por el hepatocarcinoma, la cual fue calculada como un ratio entre el número anual de las defunciones registradas (periodo 2005-2016) y el número de ciudadanos de cada departamento y región geográfica (expresados por cada 100.000 habitantes). El número de defunciones se clasificaron por el año, el sexo, los grupos de edad, el departamento y la región geográfica. Además, obtuvimos la mortalidad estandarizada por la edad mediante el método directo, utilizando como referencia a la población de la Organización Mundial de la Salud en el periodo 2000-2025. ${ }^{15}$

\section{Análisis de los datos}

El análisis descriptivo se expresó mediante las frecuencias absolutas y relativas empleando el programa Microsoft Excel $^{\circledR}$ (versión 2013 para Windows). Se realizó el cálculo del porcentaje de cambio mediante el promedio de las frecuencias de la mortalidad de los seis primeros y últimos años evaluados para reducir el sesgo de error de la medición asociado con tomar un solo año como referencia.

De igual modo, se efectuó una comparación de la distribución geográfica utilizando el software QGIS v2.10.1 (OSGeo, Beaverton, OR, EE. UU.); para ello dividimos los departamentos y regiones geográficas en quintiles de mortalidad de los años de estudio, de forma similar a un estudio previo. ${ }^{16}$

\section{Aspectos éticos}

Este estudio empleó datos secundarios que fueron obtenidas mediante un portal web de acceso público. Dicha información es anónima, por lo que no implica un riesgo directo de identificación de los sujetos.

\section{Resultados}

Características de la mortalidad por hepatocarcinoma

En el periodo 2005-2016, en la base de datos del MINSA se registraron 2170 defunciones por un hepatocarcinoma, de las cuales 1087 personas $(50,1 \%)$ eran 
de sexo masculino. La frecuencia más elevada de defunción correspondió al grupo etario mayor de 60 años con $1464(67,5 \%)$ defunciones, seguido por el grupo de $30 \mathrm{a}$ 59 años con $555(25,5 \%)$ y el de los menores de 30 años con $151(7,0 \%)$.

\section{Tendencia de la mortalidad por hepatocarci- noma}

La tasa estandarizada por edad de la mortalidad por hepatocarcinoma en Perú disminuyó de 1,1 por cada 100.000 habitantes en el 2005 a 0,7 en el 2016 (Figura 1).

La región geográfica que presentó una disminución de la mortalidad fue la de la Sierra $(\%$ de cambio $=-40,1)$.
Sin embargo, presentaron un incremento las regiones de la Selva $(\%$ de cambio $=25,5)$ y la Costa $(\%$ de cambio $=7,4)$.

Por otro lado, los departamentos con mayor disminución en la mortalidad por hepatocarcinoma fueron Huancavelica (\% de cambio $=-77,9)$, Apurímac $(\%$ de cambio $=-60,6)$ y Moquegua $(\%$ de cambio $=-56,3)$; mientras que, los departamentos que presentaron mayor incremento fueron los de Amazonas (\% de cambio $=281,9)$ y Madre de Dios (\% de cambio = 236,7). Las tasas de mortalidad más elevadas, por encima del promedio nacional en el periodo 2011-2016, fueron de los departamentos de Lambayeque, Madre de Dios y Tacna (Tabla 1 y Figura 2).

Figura 1. Tendencias de las tasas estandarizadas por edad de la mortalidad por el carcinoma de las células hepáticas en el Ministerio de Salud del Perú según el año del registro de la defunción (2005-2016)

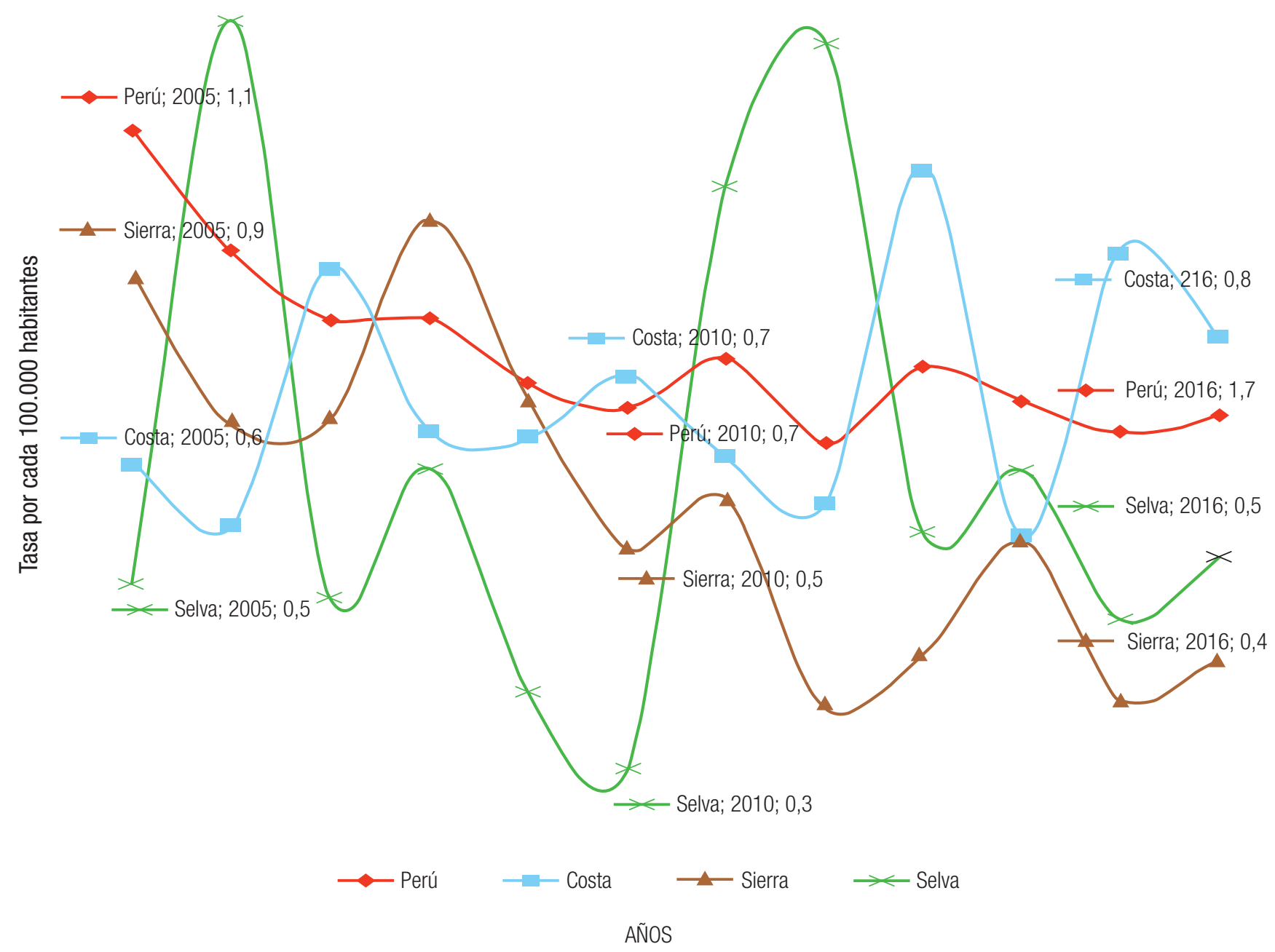

Fuente: Elaboración propia a partir del análisis de la información de la base de datos del MINSA. 
Tabla 1. Mortalidad por carcinoma de las células hepáticas registrada en el Ministerio de Salud del Perú por regiones geográficas y departamentos: comparación de los periodos 2005-2010 y 2011-2016

\section{Mortalidad x 100.000 habitantes}

\begin{tabular}{lccc} 
Período & $\mathbf{2 0 0 5 - 2 0 1 0}$ & $\mathbf{2 0 1 1 - 2 0 1 6}$ & \% de cambio \\
\hline Perú (País) & 0,84 & 0,72 & $-14,7$ \\
Región & & & \\
Costa & 0,70 & 0,75 & 7,4 \\
Sierra & 0,74 & 0,45 & $-40,1$ \\
Selva & 0,57 & 0,72 & 25,5
\end{tabular}

\section{Departamento}

\begin{tabular}{|c|c|c|c|}
\hline Amazonas & 0,16 & 0,60 & 281,9 \\
\hline Áncash & 0,30 & 0,29 & $-3,4$ \\
\hline Apurímac & 2,13 & 0,84 & $-60,6$ \\
\hline Arequipa & 0,11 & 0,20 & 75,0 \\
\hline Ayacucho & 0,99 & 0,57 & $-41,9$ \\
\hline Cajamarca & 0,47 & 0,48 & 2,8 \\
\hline Callao & 1,08 & 0,73 & $-32,8$ \\
\hline Cusco & 1,11 & 0,65 & $-41,2$ \\
\hline Huancavelica & 0,70 & 0,16 & $-77,9$ \\
\hline Huánuco & 0,33 & 0,19 & $-40,5$ \\
\hline Ica & 0,73 & 0,99 & 35,7 \\
\hline Junín & 0,95 & 0,54 & $-42,9$ \\
\hline La Libertad & 0,16 & 0,16 & 4,3 \\
\hline Lambayeque & 1,25 & 1,36 & 8,8 \\
\hline Lima & 0,75 & 0,48 & $-36,1$ \\
\hline Loreto & 0,37 & 0,30 & $-20,2$ \\
\hline Madre de Dios & 0,41 & 1,39 & 236,7 \\
\hline Moquegua & 0,64 & 0,28 & $-56,3$ \\
\hline Pasco & 0,43 & 0,39 & $-8,2$ \\
\hline Piura & 0,49 & 0,86 & 77,0 \\
\hline Puno & 0,69 & 0,54 & $-21,7$ \\
\hline San Martín & 1,10 & 0,55 & $-50,6$ \\
\hline Tacna & 0,92 & 1,74 & 88,2 \\
\hline Tumbes & 0,26 & 0,15 & $-43,0$ \\
\hline Ucayali & 0,82 & 0,76 & $-7,2$ \\
\hline
\end{tabular}

Fuente: Elaboración propia a partir del análisis de la información de la base de datos del MINSA. 
Figura 2. Distribución geográfica de las tasas estandarizadas por edad de la mortalidad (ASMR) por el carcinoma de las células hepáticas registradas en los departamentos del Perú en los periodos 2005-2010 y 2011-2016 según datos del Ministerio de Salud del Perú
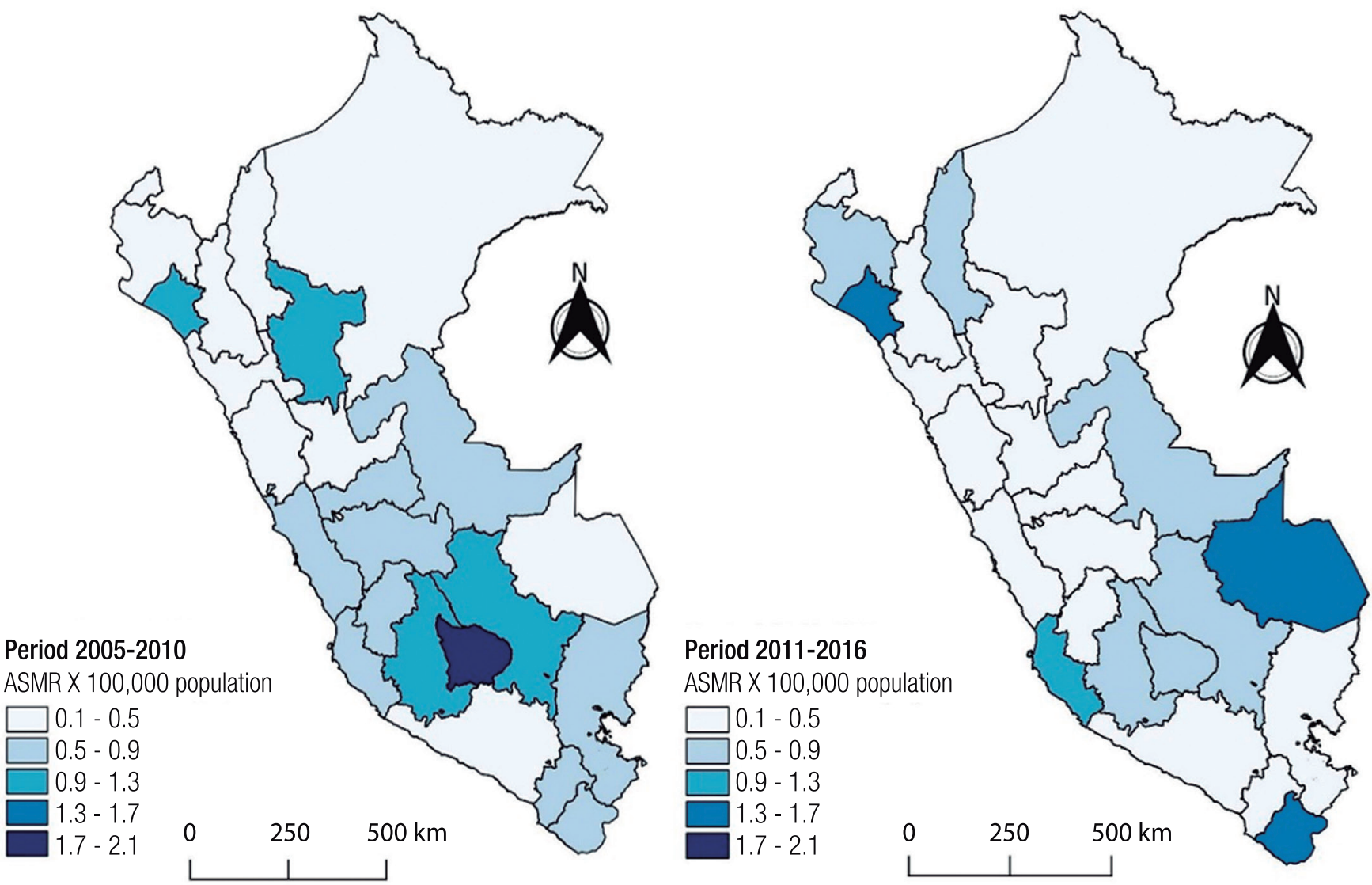

Fuente: Elaboración propia a partir del análisis de la información de la base de datos del MINSA.

\section{Discusión}

La mortalidad en el Perú por hepatocarcinoma ha llegado a niveles bastante importantes en los últimos años ocupando el segundo lugar de muerte por cáncer hasta el $2011 .^{8}$ En nuestro estudio se registraron 2170 defunciones por hepatocarcinoma desde el 2005 al 2016. El sexo masculino y la edad superior a 60 años fueron las categorías que presentaron mayor frecuencia en la mortalidad por esta neoplasia.

Si bien la tasa estandarizada por edad de la mortalidad por hepatocarcinoma hepáticas en Perú disminuyó del 1,1 por cada 100.000 habitantes en el 2005 al 0,7 en el 2016, aún notamos cifras similares y una tendencia con poca variación en los últimos 20 años. El estudio realizado en nuestro país por Farfán y Cabezas entre los años 1995 al 2000 mostró resultados similares, donde la tasa estandarizada por edad de la mortalidad cayó de 1,01 en
1995 a 0,6 en el $2000 \cdot{ }^{17}$ No se han logrado encontrar las cifras correspondientes al periodo 2001 al 2004 que nos revelen la situación de ese momento. Sin embargo, lo que sí se puede apreciar es que hubo un aumento en la tasa estandarizada por edad de la mortalidad entre los años $2000(0,6)$ y el $2005(1,1)$. Esto genera un interrogante sobre qué pasó en ese periodo de tiempo o si hubo una mejora en los registros de la mortalidad a nivel nacional.

Las características epidemiológicas identificadas en nuestro estudio son similares a las reportadas anteriormente por el resto del mundo. La mayoría de las personas fallecidas por un hepatocarcinoma eran hombres, lo cual se ha visto reportado en varios países latinoamericanos. En Chile y México la tasa de mortalidad en el 2012 fue dos veces mayor en hombres que en mujeres y en Uruguay hasta 4 veces. ${ }^{18} \mathrm{La}$ mortalidad sigue la misma tendencia que la incidencia del hepatocarcinoma, la cual es 
de 2 a 4 veces mayor en hombres que en mujeres. ${ }^{18}$ Una posible explicación se da porque los hombres tienen una mayor exposición a los factores de riesgo que pueden llevar a desarrollar el hepatocarcinoma como lo son la hepatitis viral crónica, el alcohol y el tabaquismo. ${ }^{1}$ Además, se cree que los andrógenos son responsables de que, dentro de los pacientes infectados por el virus de la hepatitis $\mathrm{B}$ (VHB) y C (VHC), los hombres sufran una mayor y más rápida progresión de la enfermedad llevándolos a sufrir complicaciones. ${ }^{1}$

Otra variable epidemiológica identificada fue una mayor mortalidad por hepatocarcinoma en los pacientes mayores de 60 años con un 67,5\%, seguido del grupo de 35 a 39 años con el 25,5\%. Este hallazgo también es percibido en estudios previos sobre la mortalidad a nivel mundial. En un trabajo realizado en EE. UU. entre los años 1988 y el 2000, la edad media de muerte en los hombres fue 61,4 y en las mujeres $65,3 .{ }^{19}$ Sin embargo, debemos mencionar que en los países con alta prevalencia del VHB, el hepatocarcinoma suele presentarse en las edades tempranas ya que el contagio se da al nacer o en la infancia. En cuanto a zonas donde existe una mayor cantidad del VHC la infección se da más tardíamente y, por lo tanto, las complicaciones también son más lentas. Además, a medida que los pacientes envejecen, se le van añadiendo otros factores de riesgo que contribuyen a desarrollar un hepatocarcinoma. ${ }^{1}$ Nuestro país vive una situación difícil, ya que no solo tenemos áreas de alta endemicidad por el $\mathrm{VHB}$, sino que incluso existen zonas donde el VHC va en aumento. ${ }^{10,20}$ Esto hace que exista una alta tasa de mortalidad, no solo en el grupo de los mayores de 60 años, sino que se evidencie un aumento en el grupo de 35 a 39 años en comparación con años anteriores. ${ }^{17}$

Con respecto a las regiones geográficas afectadas, hubo una disminución en la tasa de mortalidad en la Sierra (\% de cambio $=-40,1)$, mientras que las regiones de la Selva y la Costa sufrieron aumentos del 25,5 y el 7,4\% respectivamente. En la Sierra, la mayor disminución se presentó en Huancavelica, Apurímac y Moquegua. Estos resultados son similares al estudio publicado por Ramírez Soto et al sobre la mortalidad en Apurímac donde se evidencia una TME que ha ido descendiendo desde el $2007(6,9)$ al $2010(3,8) \cdot{ }^{11}$ Esto se ha logrado a través de programas de prevención y control del VHB encabezados por el programa de vacunación universal para los menores de un año y los grupos de riesgo. ${ }^{20}$ Además, se creó la vigilancia centinela del síndrome ictérico agudo y la seroprevalencia del antígeno de la hepatitis B. ${ }^{20}$ Sin embargo, la Selva presentó aumentos de más del 200\% en Amazonas y Madre de Dios. Como se aprecia en los estudios previos, Madre de
Dios sigue encabezando la lista de mayor mortalidad por el hepatocarcinoma desde el año 2000 donde la TME fue del 7,11. ${ }^{17}$ Un punto a evaluar más adelante es el acceso a los servicios de salud en estos lugares y las estrategias de prevención que presentan. Un análisis sistemático en los EE. UU. demostró que los pacientes que son diagnosticados de hepatocarcinoma, gracias a un sistema de vigilancia para el cáncer, son capaces de recibir un tratamiento potencialmente curativo reduciendo así su mortalidad. ${ }^{21}$ Asimismo, en China, dos ensayos controlados aleatorizados demostraron que la tasa de supervivencia a 1 y 2 años por hepatocarcinoma en pacientes portadores de VHB diagnosticados en estadios iniciales a través del screening fue del $88 \%$ y del $77,5 \%$ respectivamente versus el $0 \%$ en los pacientes sin vigilancia. ${ }^{22}$

El presente estudio tiene limitaciones que deben ser consideradas. El análisis realizado fue en base a los certificados de defunción que son llenados por los distintos profesionales de la salud a nivel nacional. Estos podrían presentar errores por la falta de conocimiento sobre la forma correcta de completarlos o por la falta de tiempo. Según la OMS nuestro país se encuentra dentro del rango de baja calidad para llenar los certificados de defunción. ${ }^{23}$ Esto podría resultar en un sesgo de la medición. Otra limitación identificada aparece al utilizar datos anónimos que no permiten obtener mayores factores de riesgo $\mathrm{u}$ otros datos de las historias clínicas que nos posibilitarían un análisis más detallado. Además, existen estudios limitados en nuestro país sobre este tema, por lo que es difícil realizar contrastes y comparar cifras. Sin embargo, este análisis, ante la falta de información estadística reciente y con una mortalidad considerable, sirve para actualizar la información importante sobre esta neoplasia y expone la necesidad de continuar profundizando en este tema.

En conclusión, la mortalidad estandarizada por edad del hepatocarcinoma en el Perú presenta una disminución desde el 2005 al 2016; no obstante, en los últimos años la variación ha sido mínima. Es importante recalcar, además, que la mortalidad causada por el hepatocarcinoma parece no haber cambiado demasiado desde 1995 al 2000. Los varones mayores de 60 años fallecen más que otros grupos y la mayor tasa de mortalidad se encuentra en la Selva.

Hasta el momento, no hemos encontrado cifras actualizadas a nivel nacional sobre esta patología. Se sugiere desarrollar estudios longitudinales analíticos que puedan complementar esta información.

Conflicto de intereses. Todos los autores niegan cualquier tipo de conflicto de interés. Estudio autofinanciado. 


\section{Referencias}

1. Wallace MC, Preen D, Jeffrey GP, Adams LA. The evolving epidemiology of hepatocellular carcinoma: a global perspective. Expert Rev Gastroenterol Hepatol 2015; 9 (6): 765-779. doi: 10.1586/17474124.2015.1028363.

2. World Cancer Research Fund/American Institute for Cancer Research. Continuous Update Project Expert Report 2018. Diet, nutrition, physical activity and liver cancer 2015 (Revised 2018). Disponible en: https:/www.wcrf.org/sites/default/files/Liver-cancer-report.pdf

3. Howlader N, Noone AM, Krapcho M, Miller D, Brest A, Yu M, Ruhl J, Tatalovich Z, Mariotto A, Lewis DR, Chen HS, Feuer EJ, Cronin KA. SEER Cancer Statistics Review, 1975-2016, National Cancer Institute. Bethesda 2019.

4. Ascione A, Fontanella L, Imparato M, Rinaldi L, De Luca M. Mortality from cirrhosis and hepatocellular carcinoma in Western Europe over the last 40 years. Liver Int 2017; 37 (8): 1193-1201. doi: 10.1111/liv.13371.

5. Forner A, Reig M, Bruix J. Hepatocellular carcinoma. Lancet 2018; 391 (10127): 1301-1314. doi: 10.1016/S01406736(18)30010-2.

6. Payet Meza E, Pérez Mejía P, Poquioma Rojas E, Díaz Nava E. Registro de cáncer de Lima Metropolitana. Incidencia y mortalidad 2010-2012. Instituto Nacional de Enfermedades Neoplásicas 2016. Volumen 5. Disponible en: http://bvs.minsa.gob.pe/local/ MINSA/3774.pdf

7. Bustíos Sánchez C, Díaz Ferrer J, Román Vargas R, Dávalos Moscol M, Zumaeta Villena E. Características clínico - epidemiológicas del carcinoma hepatocelular y su tratamiento en el departamento del aparato digestivo del HNERM ES-SALUD. Rev Gastroenterol Perú 2009; 29: 17-23. PMID: 19424404.

8. Ramos WC, Venegas DR. Análisis de la situación del cáncer en el Perú. Ministerio de Salud del Perú; Dirección General de Epidemiología 2013. Disponible en: https://www.dge.gob.pe/portal/ docs/asis_cancer.pdf

9. Yang JD, Hainaut P, Gores GJ, Amadou A, Plymoth A, Roberts LR. A global view of hepatocellular carcinoma: trends, risk, prevention and management. Nat Rev Gastroenterol Hepatol 2019; 16 (10): 589-604. doi: 10.1038/s41575-019-0186.

10. Montes Teves P. Hepatitis C: retos pendientes. Rev Gastroenterol Perú 2014; 34 (3): 187-188. PMID: 25293985.

11. Ramírez-Soto M, Guevara-Cuadros J, Huichi-Atamari M. Mortalidad por Hepatocarcinoma y cirrosis hepática en Apurímac: 2007-2010. Rev Peru Epidemiol 2012; 16: 1-5.
12. Cabezas-Sánchez C, Trujillo-Villarroel O, Zavaleta-Cortijo C, Culqui-Lévano D, Suarez-Jara M, Cueva-Maza N, Monzón S. Prevalencia de la infección por el virus de hepatitis B en niños menores de 5 años de comunidades indígenas de la Amazonía peruana posterior a intervenciones mediante inmunización. Rev Peru Med Exp Salud Pública 2014; 31 (2): 204-210. PMID: 25123856.

13. Nelson NP, Easterbrook PJ, McMahon BJ. Epidemiology of Hepatitis B Virus Infection and Impact of Vaccination on Disease. Clin Liver Dis 2016; 20 (4): 607-628. doi: 10.1016/j. cld.2016.06.006.

14. Vildozola Gonzáles H. Hepatitis B y vacunación. Acta Med peruana 2008; 25 (2): 57.

15. Ahmad OB, Boschi-Pinto C, Lopez AD, Murray CJL, Lozano R, Inoue M. Age Standardization of Rates: A New WHO Standard. GPE Discussion Paper Series: No.31. World Health Organization 2001. Disponible en: https://www.who.int/healthinfo/paper31.pdf?ua=1

16. Atamari-Anahui N, Ccorahua-Rios MS, Taype-Rondan A, Mejia CR. Mortalidad atribuida a diabetes mellitus registrada en el Ministerio de Salud de Perú, 2005-2014. Rev Panam Salud Publica 2018; 42: e50. doi: 10.26633/RPSP.2018.50

17. Farfán G, Cabezas C. Mortalidad por enfermedades digestivas y hepatobiliares en el Perú, 1995-2000. Rev gastroenterol Perú 2002; 22 (4): 310-323. PMID: 12525847.

18. Bertuccio P, Turati F, Carioli G, Rodriguez T, La Vecchia C, Malvezzi M, Negri E. Global trends and predictions in hepatocellular carcinoma mortality. J Hepatol 2017; 67 (2): 302-309. doi: 10.1016/j.jhep.2017.03.011.

19. Ray Kim W, Gores GJ, Benson JT, Therneau TM, Melton LJ. Mortality and hospital utilization for hepatocellular carcinoma in the United States. Gastroenterol 2005; 129 (2): 486-493. doi: 10.1016/j.gastro.2005.05.001.

20. Cabezas C. Hepatitis viral B y Delta en el Perú: epidemiología y bases para su control. Rev Peru Med Exp Salud Publica 2007; 24 (4):378-397.

21. Singal AG, Pillai A, Tiro J. Early detection, curative treatment, and survival rates for hepatocellular carcinoma surveillance in patients with cirrhosis: a meta-analysis. PLoS Med 2014; 11 (4): e1001624. doi: 10.1371/journal.pmed.1001624.

22. Kanwal F, Singal AG. Surveillance for Hepatocellular Carcinoma: Current Best Practice and Future Direction. Gastroenterology 2019; 157 (1): 54-64. doi: 10.1053/j.gastro.2019.02.049.

23. Mathers CD, Fat DM, Inoue M, Rao C, Lopez AD. Counting the dead and what they died from: an assessment of the global status of cause of death data. Bull World Health Organ 2005; 83 (3): 171-177. PMID: 15798840. 